\title{
Could Phlebotomus mascittii play a role as a natural vector for Leishmania infantum? New data
}

\author{
Adelheid G. Obwaller ${ }^{1,2}$, Mehmet Karakus ${ }^{3,5}$, Wolfgang Poeppl ${ }^{4}$, Seray Töz ${ }^{3}$, Yusuf Özbel ${ }^{3}$, Horst Aspöck²
} and Julia Walochnik ${ }^{2^{*}}$

\begin{abstract}
Background: The occurrence of phlebotomine sand flies in Central Europe was questioned until they were recorded for the first time in Germany in 1999, and ten years later also in Austria. The aim of this study was to investigate sand flies collected in Austria for their carrier status of Leishmania spp.

Findings: From 2012 to 2013 field studies were conducted in eastern Austria. Altogether, 22 individuals of sand flies were found, all morphologically identified as Phlebotomus (Transphlebotomus) mascittii Grassi, 1908. Twelve non-engorged female specimens with no visible remnants of a blood meal in their bodies were individually investigated for Leishmania spp. by ITS-1 real-time PCR. One out of these was positive for Leishmania, identified as Leishmania infantum by DNA sequencing. This finding suggests that L. infantum is not excreted by $P$. mascittii and possibly can establish an infection within P. mascittii. Interestingly, an asymptomatic dog living on the farm where this sand fly had been caught was also Leishmania-positive.

Conclusions: This study provides new data on the suspected vector capacity of $P$. mascittii, being the northernmost sand fly species in Europe and in most central European regions the only sand fly species found. Proven vector capacity of $P$. mascittii for Leishmania spp. would be of significant medico-veterinary importance, not only with respect to expanding sand fly populations in Central Europe related to global warming, but also in the light of globalization and increasing movements of humans.
\end{abstract}

Keywords: Phlebotomine sand fly, Phlebotomus mascittii, Leishmania infantum, Natural infection, Central Europe, Austria

Abbreviations: $\mathrm{CL}$, Cutaneous leishmaniasis; ITS-1, Internal transcribed spacer 1; MCL, Mucocutaneous leishmaniasis; PCR, Polymerase chain reaction; VL, Visceral leishmaniasis; WHO, World Health Organisation

\section{Background}

Leishmaniasis is a sand fly-borne disease caused by intracellular protozoan parasites of the genus Leishmania, with at least 15 species being pathogenic for humans [1]. According to the World Health Organisation, 98 countries, mostly in tropical and subtropical regions, are considered endemic, with 350 million people living at risk of infection and estimated 1.3 million new cases and 20,000-30,000 deaths annually [2]. The clinical spectrum comprises visceral leishmaniasis

\footnotetext{
* Correspondence: julia.walochnik@meduniwien.ac.at

${ }^{2}$ Institute of Specific Prophylaxis and Tropical Medicine, Medical University of Vienna, Kinderspitalgasse 15, 1090 Vienna, Austria

Full list of author information is available at the end of the article
}

(VL) and several different forms of cutaneous (CL) and mucocutaneous leishmaniases (MCL), but infections can also remain entirely asymptomatic. Leishmania spp. are transmitted by female phlebotomine sand flies (Diptera: Psychodidae: Phlebotominae). Among more than 800 recognized species of sand flies, 78 species are proven vectors of Leishmania spp. [3].

The two endemic European transmission cycles include zoonotic VL and CL caused by $L$. infantum throughout all southern countries of Europe and anthroponotic CL caused by L. tropica sporadically occurring in Greece. Moreover, $L$. donovani, causing VL and CL, has been recently introduced to Cyprus [4]. In the Mediterranean region, 1200-2000 human cases of VL and 239,500-393,600 
cases of CL are estimated to occur annually [5], with a multiple of asymptomatic infections estimated to occur per each symptomatic case [6]. In southern Europe, dogs are the main reservoirs of $L$. infantum [7]. The principal vectors for $L$. infantum in the Mediterranean area are Phlebotomus species of the subgenera Larroussius (P. ariasi, $P$. neglectus, $P$. kandelaki, $P$. perfiliewi, $P$. perniciosus and P. tobbi) and Adlerius (P. balcanicus), and $P$. (Paraphlebotomus) sergenti and $P$. (Phlebotomus) papatasi are considered the main vectors of $L$. tropica and $L$. major, respectively, in this region $[3,4]$.

Since the early reports from Switzerland and France [8-10], the occurrence of phlebotomine sand flies north of the Alps and their possible medical relevance has been a matter of debate. Further proof for autochthonous sand fly populations in Central Europe came with records of Phlebotomus (Transphlebotomus) mascittii from Belgium, France, Germany and later also Austria [7, 11-13]. P. mascittii was first described by Grassi in 1908 from Rome (Italy), and generally appears to be widespread in Europe, albeit in low population densities and with low rates of biting humans [7]. The vector competence of $P$. mascittii has not been conclusively clarified so far, but $P$. mascittii has been described as a generally aggressive species, has been reported to suck blood on humans [10] and a few assumedly autochthonous cases of leishmaniasis in human and animals have been described in regions where $P$. mascittii is the only sand fly species found, including two cases in Austria (reviewed in [14-16]). Moreover, one female specimen of P. mascittii was found positive for Leishmania spp. by polymerase chain reaction (PCR) on the island of Montecristo, Italy [17].

In Austria, P. mascittii was first recorded in 2009 [12]. Since then, several field studies in various regions of Austria have been conducted $[16,18]$, and interestingly, P. mascittii was only found close to human dwellings, usually farms with livestock and companion animals, corroborating its adaption to man-made environments. The aim of the present study was to investigate Austrian specimens of $P$. mascittii for their carrier status for Leishmania spp.

\section{Methods}

\section{Study area and sand fly sampling}

The sampling site was located in eastern Austria, in the federal state of Lower Austria, around $16 \mathrm{~km}$ (linear distance) from the border with Slovakia and $21 \mathrm{~km}$ from the border with Hungary (Fig. 1). The area is characterised by a Pannonian climate with continental influence, with hot summers and cold winters. The entomological field studies were performed in the village of Rohrau (48 $33^{\prime} 57^{\prime \prime} \mathrm{N}, 1^{\circ} 51^{\prime} 33^{\prime \prime} \mathrm{E}$; altitude $148 \mathrm{~m}$ ) in July and August 2012 and 2013 using 440 light traps in total. At this site, sand flies had already been found before.

Sand flies were trapped and identified as described previously $[16,18]$. In brief, we used battery-operated

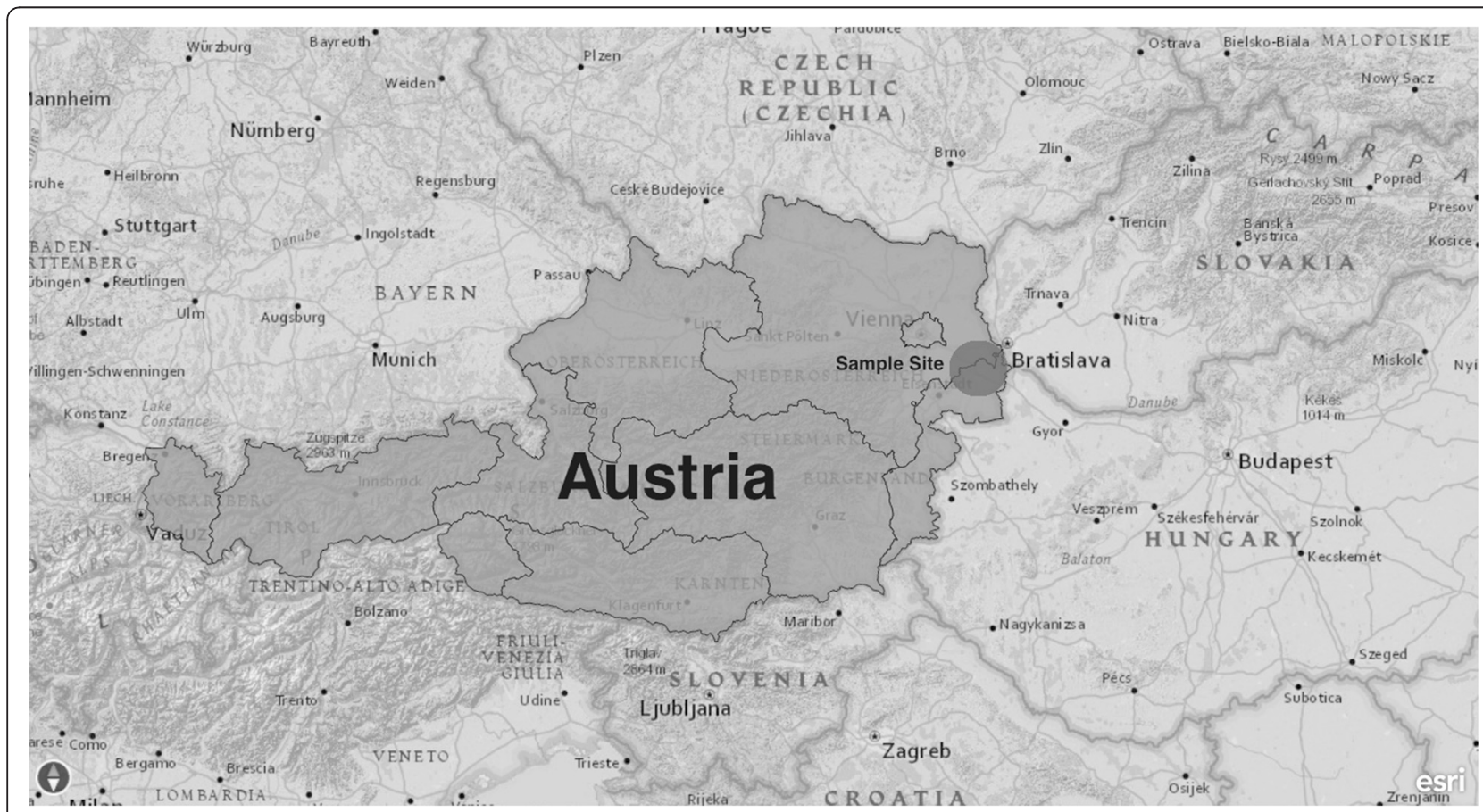

Fig. 1 Map showing geographical location of the study area 
CDC Miniature Light Traps, with an ultra-fine mesh (John W. Hock Company, Gainesville, FL, USA). Sand flies were collected with up to eight traps distributed at the sampling site, a farmhouse with a nineteenth century partially open wooden barn with loamy ground and an open garage with concrete pavement. Horses, several cats and a dog were held on the farm. The trapped sand flies were transferred into $70 \%$ ethanol for further investigation. For identification, specimens were cleared with potassium hydroxide solution and slide-mounted in Hoyer's fluid. Species identification was based on basic taxonomical structures, mainly, pharynx, male genitalia and female spermathecae using the identification keys by Theodor and Seccombe et al. $[19,20]$.

\section{DNA extraction and PCR assays}

Males and fed females were excluded from the analysis. All non-engorged female specimens of $P$. mascittii were prepared as follows: thorax and abdomen of the sand flies were individually transferred to ZR Bashing Bead ${ }^{\mathrm{mx}}$ tubes (Zymo Research Corporation, Freiburg, Germany) and homogenised using a MagNa Lyser ${ }^{\circ}(7000$ rpm 90 s, Roche Molecular Diagnostics, Mannheim, Germany). Male sand fly specimens, a blood sample from a healthy dog and high pure PCR water were used as negative controls. Tubes were incubated overnight at $56{ }^{\circ} \mathrm{C}$ after adding $500 \mu \mathrm{l}$ volume of Qiagen ${ }^{\circ}$ Tissue lysis buffer (Qiagen Inc., Vienna, Austria). DNA extractions were made using the Qiagen ${ }^{\circ}$ Tissue\&Blood extraction kit with a slight modification on the final elution step (elution in a volume of $50 \mu \mathrm{l}$ ) to obtain high yield of DNA.

Real-time PCR targeting the internal transcribed spacer 1 (ITS-1) region between the $18 \mathrm{~S}$ and the $5.8 \mathrm{~S}$ rRNA genes was performed using species-specific primers for detecting and distinguishing $L$. donovani/infantum complex, L. tropica and L. major [21]. PCR was performed using 20 ng genomic DNA, 10 pmol of each primer, 200 $\mathrm{nM}$ of each probe and $20 \mu \mathrm{l}$ QuantiTect $^{\circ}$ Probe PCR mix per reaction. Melting curves were generated using channel 2 and 3. Three international reference strains $L$. infantum (MHOM/TN/80/IPT1), L. tropica (MHOM/ SU/74/SAF-K27) and L. major (MHOM/SU/73/5ASKH) were included to obtain standard curves.

A conventional PCR, also targeting the ITS-1 region and using the LITSR/L5.8S primers [22], was performed with a ready-to-use PCR master mix (Helixamp ${ }^{\circ} \mathrm{T} 500 \mathrm{~N}$ ) to obtain sequence data for the positive sample. PCR products were visualized in a $1.5 \%$ agarose gel using a UView $^{\text {ma }}$ Mini Transilluminator. The amplicon was purified using a PCR band extraction kit (QIAquick ${ }^{\circ}$, Qiagen) and sequenced commercially. Sequence data were analysed using Geneious R8 [23].
At the time the sand flies were caught, also an EDTA whole blood sample of the dog living at the collection site was taken and also tested for Leishmania spp. by conventional ITS-1 PCR as described above. The DNA was extracted from the blood using the peqGOLD Blood DNA Mini Kit (Peqlab, Erlangen, Germany). After PCR, the amplicon was purified and sequenced in both directions using the BigDye ${ }^{\odot}$ Terminator v1.1 Cycle Sequencing Kit and an automatic 310 ABI PRISM sequencer (Applied Biosystems, Darmstadt, Germany).

As, according to the owners, the approximately oneyear-old male dog had never been outside of Austria, EDTA whole blood samples were also obtained from a sibling and the mother bitch, both living in a distant region of Austria and never having been to the farm. These samples were also tested by Leishmania-specific PCR as described above. Unfortunately, the samples from the mother bitch and the sibling were lost for follow-up by DNA sequencing. All sequence data obtained were submitted to the GenBank database under accession numbers KT026221 (sand fly) and KU555887 (dog).

\section{Results and discussion}

A total of 22 sand flies were trapped during the study period, namely six females and one male in 2012 and 13 females and two males in 2013. All specimens were identified as Phlebotomus (Transphlebotomus) mascittii, the only species known to occur in Austria so far. Altogether, 12 female specimens were un-engorged with no visible remnants of a blood meal in their bodies. Out of these, one specimen (specimen No. 9) showed a $L$. donovani/infantum complex-specific melting peak in the ITS-1 real time PCR, with high analogy to the positive control for L. infantum (MHOM/TN/80/IPT1) (Fig. 2). The three negative controls and the remaining specimens did not reveal any peaks. As this real time PCR cannot identify below the level of $L$. donovani/infantum complex, we conducted a conventional ITS-1 PCR to obtain sequence data. The obtained sequence from the sand fly showed a $100 \%$ similarity (315/315 bp) to numerous strains of L. infantum (e.g. KC477100), including also the strain obtained from the dog living on the farm where the Leishmania-positive sand fly had been caught. According to the owners, the approximately 1-year-old male dog had never been outside of Austria. He had lived one transmission season on the farm, spending most of the nights in the open and having access to the barn where the infected sand fly was caught. Our working hypothesis is that the sand fly took up the parasites during a blood meal on the L. infantum-positive dog and that the dog had acquired its infection by vertical transmission from the bitch living in a distant farm in Austria. The mother bitch and one of the siblings were 


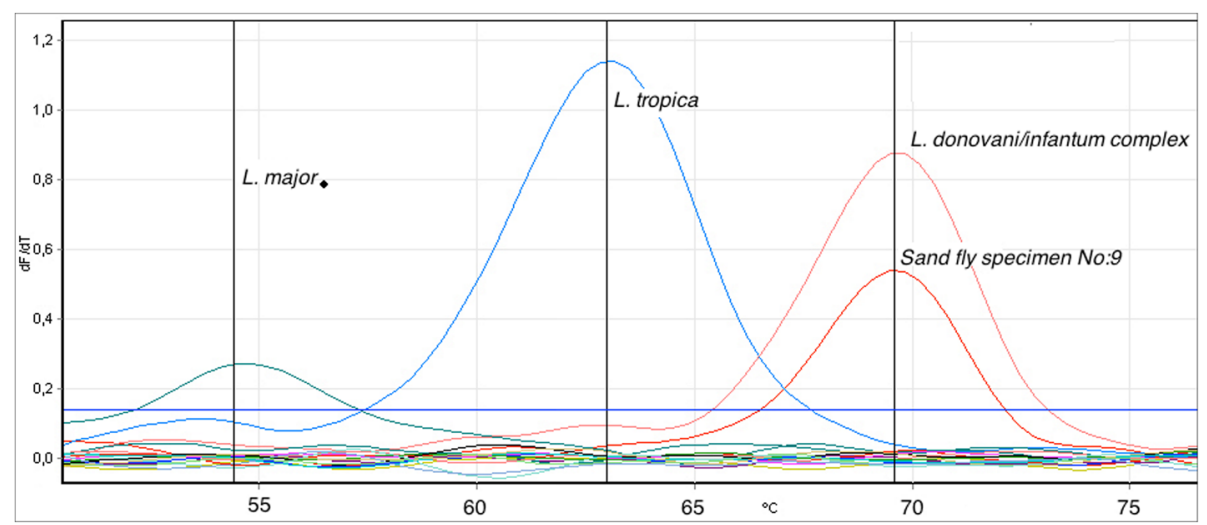

Fig. 2 Melting temperature analysis showing the specific melting peak for the L. donovani/infantum complex (negative specimens including controls are under the threshold in the graph)

also positive for Leishmania spp. by PCR and an independent infection seems implausible. The possibility of vertical transmission in canine leishmaniasis has been reported previously [24, 25]. The bitch was used for breeding and had travelled abroad frequently. However, how exactly the bitch became infected cannot be deduced unequivocally; veneral transmission should also be taken into account [25].

The presence of Leishmania DNA in P. mascittii was first reported from the Island of Montecristo, Italy, in 2014, albeit without details on the feeding status of the respective sand fly [17]. The current study, demonstrating Leishmania DNA in an un-engorged specimen of $P$. mascittii, now provides further data on the possible vector capacity of $P$. mascittii, the northernmost sand fly species in Europe, and in most central European regions the only sand fly species found. The established criteria for the incrimination of natural vectors are: (i) anthropophilic behaviour; (ii) repeated isolation of the same species of Leishmania from the sand fly as that found in patients; (iii) feeding on reservoir host(s); (iv) development of the parasite in the sand fly; and (v) ability to transmit the parasite by bite to a susceptible host while taking a blood meal [26]. For P. mascittii, as outlined in the introduction, the first three of these criteria essentially apply. Our data now suggest that L. infantum is at least not excreted with the faeces by $P$. mascittii and might thus be able to establish a long-term infection in P. mascittii.

Indicative data for this assumption firstly is that the Leishmania-positive sand fly was a clearly un-engorged specimen. In sand flies held at $28{ }^{\circ} \mathrm{C}$, the blood meal is typically digested within $72 \mathrm{~h}$, having a bright red colour during the first $18 \mathrm{~h}$, a dark red colour during 18-24 h and a brown colour between 24 and $72 \mathrm{~h}$ [27]. At lower temperatures, defecation is delayed. In a study, in which sand flies were held at $26{ }^{\circ} \mathrm{C}$, the majority of sand flies had lost their blood by days $7-8$, only $14-27 \%$ of them still showing remnants of a blood meal [28]. Lower temperatures however, even as low as $20^{\circ} \mathrm{C}$, do not prohibit but only delay Leishmania development in sand flies [29]. In the current study, no remnants of a blood meal were visible, thus the blood meal must have occurred at least $72 \mathrm{~h}$, but because of day temperatures around $24^{\circ}$ $\mathrm{C}$ and night temperatures around $19{ }^{\circ} \mathrm{C}$ [18], rather $\geq$ 8 days before the trapping. This suggests that the parasites might indeed have established an infection in the sand fly, because otherwise, the parasites would have been defecated with the blood remnants and no DNA would have been detectable. DNA of non-living cells is known to be rapidly degraded in the sand fly gut, e.g. at $28^{\circ} \mathrm{C}$ the DNA of cells of the blood host can only be detected until $24 \mathrm{~h}$ after engorgement, already after $37 \mathrm{~h}$ no host cell DNA is detectable any more [27]. This rapid degradation of DNA is explained by a peak activity of proteases and DNAses between 24 and 48 h after feeding in the sand fly gut [27], which however of course, is also temperature-dependent. Moreover, it is unknown whether the DNA in dying or dead Leishmania cells behaves similarly to DNA in dying or dead host cells and amastigote Leishmania DNA is not only protected by the Leishmania cell but also by the host cell and might thus be detectable for longer periods. In a competent vector, the development of Leishmania spp. proceeds in the digestive tract, the precise location differing between species of the subgenera Leishmania and Viannia. Leishmania infantum is a suprapylarian parasite, developing in the midgut of the sand fly. Surviving the early phase after uptake by the sand fly is the first hurdle parasites must overcome [30]. Many Leishmania species are very specifically linked to a certain sand fly species, e.g. $L$. tropica is specifically adapted to $P$. sergenti and $P$. sergenti has been shown to be refractory to $L$. major and L. donovani because these two species cannot 
specifically bind to the midgut epithelium of $P$. sergenti and are thus defecated [28]. The latter study also demonstrated that the loss of infection with $L$. major and $L$. donovani in $P$. sergenti correlates with the excretion of the digested blood meal, which in that study was completed at approximately day 8 after the blood meal. According to Pimenta et al. [31], up to $50 \%$ of the parasites are killed by secreted proteolytic enzymes and excreted during the first day after the blood meal, even in proven vectors. The parasites must then, to establish an infection in the sand fly, attach to the midgut epithelia via specific lipophosphoglycans, constituting an assumed determinant of parasite-vector specificity [28, 30]. In Lutzomyia migonei, which has recently been recognized as a permissive vector for $L$. infantum, metacyclic promastigotes develop within five days after the blood meal [32]. In P. perniciosus, L. infantum starts to proliferate two days after the blood meal and develops heavy late-stage infections within 8 days even at only $20{ }^{\circ} \mathrm{C}$ ambient temperature [29]. The missing proof of metacyclic promastigotes from the infected sand fly is the major limitation of the current study. However, the isolation and culture of Leishmania spp. from dissected wild-caught sand flies is difficult, the prevalence even in proven vectors sometimes is low and thus large numbers of individuals are required. In an Italian study, only one out of 70 dissected sand flies revealed microscopically detectable promastigotes, but $47 \%$ of the sand flies investigated by nested PCR were positive for Leishmania spp. [33]. In Austria, the sand fly populations are scattered and extremely small. In the current study we only had 12 un-engorged and seven engorged female specimens available and the entire thorax and abdomen of the sand flies were used for DNA isolation to be as sensitive as possible, as is common practice.

A second valid argument for the possible ability of $L$. infantum to establish within $P$. mascittii is, that $L$. infantum generally seems to have a particular predisposition to be transmitted by multiple vectors. It is the only Leishmania species known that can be transmitted by sand flies of the genus Phlebotomus as well as by sand flies of the genus Lutzomyia. Recently, it has been shown, that not only Lutzomyia longipalpis but also $L$. migonei is a permissive vector for $L$. infantum [31]. Thus, it seems conceivable, that this species can also accept various species within the genus Phlebotomus as vectors. In southern European countries, proven vectors of $L$. infantum are $P$. ariasi, $P$. perniciosus and $P$. neglectus, but up to 13 more species are considered suspected vectors $[1,26]$.

Altogether, if $P$. mascittii should really prove to be a permissive vector for $L$. infantum, this would be of significant medical relevance for central Europe, as parasite reservoirs are already given in most regions. In Austria, a study by Poeppl et al. [34] revealed an unexpected high (4.5\%) seropositivity against Leishmania spp. in 1048 asymptomatic Austrians, most, of course, with travel history, but two being also PCR-positive, thus potential resevoirs. More importantly, increasing numbers of dogs in Central Europe are infected with Leishmania spp., firstly because travelling with animals has become easier since the opening of borders, but secondly also because dogs from endemic areas are sold over the internet and stray dogs are relocated from endemic areas by animal lovers. In 2008, Leschnik et al. [35] examined 119 Austrian symptomatic dogs that had been abroad, 55 of them being positive for Leishmania spp. Germany is estimated to have several thousands of infected dogs, the majority coming from Italy, Spain and Portugal [25]. To date, the population sizes of sand flies in Central Europe, including Austria, are still extremely small, but increases in population densities can be expected. In the exceptionally hot summer of 2015 we collected almost as many sand flies in two trapping nights, as we had collected the years before in over 700 trapping nights (unpublished data).

\section{Conclusions}

This study provides new data on the possible vector role of $P$. mascittii for L. infantum. Phlebotomus mascittii is the northernmost sand fly species in Europe and in most central European regions the only species found. With currently growing Leishmania reservoirs in previously non-endemic regions due to travelling, import and transport of dogs and large-scale movements of people, the evidence for vector capacity of $P$. mascittii would be of high medical importance, particularly as sand fly populations in Central Europe can be expected to expand with global warming.

\section{Acknowledgments \\ The authors wish to thank Iveta Häfeli from the Institute of Specific Prophylaxis and Tropical Medicine, Medical University of Vienna, for excellent technical assistance. \\ Funding \\ This work was supported by the Austrian Federal Ministry of Defence and Sports, Division of Science, Research and Development and the Medical University of Vienna, Austria. The funders had no role in study design, data collection and analysis, decision to publish or preparation of the manuscript.}

\section{Availability of data and materials}

Sequence data were submitted to GenBank and are available under the following accession numbers: KT026221 (sand fly); KU555887 (dog).

\section{Authors' contributions}

Conceived the study and were involved in sampling, molecular analyses and writing the manuscript: AGO, WP, HA, JW. DNA isolation from the sand flies, did part of the molecular work and also helped to prepare the manuscript: MK, ST, YÖ. All authors read and approved the final version of the manuscript.

Competing interests

The authors declare that they have no competing interests. 


\section{Consent for publication}

Not applicable.

\section{Ethics approval and consent to participate}

Informed consent for taking the respective singular blood samples from the dogs was obtained from the respective dog owners.

\section{Author details}

'Division of Science, Research and Development, Federal Ministry of Defence and Sports, Rossauer Laende 1, 1090 Vienna, Austria. ${ }^{2}$ Institute of Specific Prophylaxis and Tropical Medicine, Medical University of Vienna, Kinderspitalgasse 15, 1090 Vienna, Austria. ${ }^{3}$ Department of Parasitology, Ege University Faculty of Medicine, 35100 Bornova, Izmir, Turkey. ${ }^{4}$ Department of Dermatology, Medical University of Vienna, Waehringer Guertel 18-20, 1090 Vienna, Austria. ${ }^{5}$ Near East University, Faculty of Science and Letters, Molecular Biology and Genetics, Nicosia, Cyprus.

\section{Received: 29 March 2016 Accepted: 11 August 2016}

\section{Published online: 19 August 2016}

\section{References}

1. Akhoundi M, Kuhls K, Cannet A, Votýpka J, Marty P, Delaunay P, Sereno D. A historical overview of the classification, evolution, and dispersion of Leishmania parasites and sandflies. PLoS Negl Trop Dis. 2016;10(3):e0004349. doi:10.1371/journal.pntd.0004349.

2. WHO. Leishmania fact sheet number 375. 2016. http://www.who.int mediacentre/factsheets/fs375/en/. Accessed 11 Feb 2016

3. Alten B, Maia C, Afonso MO, Campino L, Jiménez M, González E, et al. Seasonal dynamics of phlebotomine sand fly species proven vectors of Mediterranean leishmaniasis caused by Leishmania infantum. PLoS Negl Trop Dis. 2016;10(2):e0004458. doi:10.1371/journal.pntd.0004458.

4. Antoniou M, Gramiccia M, Molina R, Dvorak V, Volf $P$. The role of indigenous phlebotomine sand flies and mammals in the spreading of leishmaniasis agents in the Mediterranean region. Euro Surveill. 2013;18(30):1-8.

5. Alvar J, Vélez ID, Bern C, Herrero M, Desjeux P, Cano J, Jannin J den Boer M WHO Leishmaniasis Control Team. Leishmaniasis worldwide and global estimates of its incidence. PLoS One. 2012:7(5):1-12. doi:10.1371/journal. pone.0035671

6. Dujardin JC, Campino L, Cañavate C, Dedet JP, Gradoni L, Soteriadou K, et al. Spread of vector-borne diseases and neglect of leishmaniasis, Europe. Emerg Infect Dis. 2008;14(7):1013-8. doi:10.3201/eid1407.071589.

7. Ready PD. Leishmaniasis emergence in Europe. Euro Surveill. 2010;15(10):1-13.

8. Galli-Valerio B. Beobachtungen über Culiciden und Mitteilung über das Vorkommen von Phlebotomus papatasi (Scop.) im Kanton Waadt. Zentralb Bakteriol Mikrobiol Hyg [A]. 1912;43:222

9. Gaschen H. Présence de Phlebotomus mascittii Grassi 1908 dans le canton de Vaud. Mitt Schweiz Entomol Ges. 1956;29:223-5.

10. Callot J. Présence de Phlebotomus larroussei en Alsace. Ann Parasitol Hum Comp. 1950;25:112.

11. Depaquit J, Naucke TJ, Schmitt C, Ferté H, Léger N. A molecular analysis of the subgenus Transphlebotomus Artemiev, 1984 (Phlebotomus, Diptera, Psychodidae) inferred from ND4 mtDNA with new northern records of Phlebotomus mascittii Grassi, 1908. Parasitol Res. 2005:95:113-6. doi:10.1007/s00436-004-1254-x.

12. Naucke TJ, Lorentz S, Rauchenwald F, Aspöck H. Phlebotomus (Transphlebotomus) mascittii Grassi, 1908, in Carinthia: First record of the occurrence of sand flies in Austria (Diptera: Psychodidae: Phlebotominae). Parasitol Res. 2011;109:1161-4. doi:10.1007/s00436-011-2361-0.

13. Kasbari M, Rave C, Noel H, Pesson B, Schaffner F, Depaquit J. Possibility of leishmaniasis transmission in Jura, France. Emerg Infect Dis. 2012;18(6):10301. doi:10.3201/eid1806.120158.

14. Pesson B, Leger N, Madulo-Leblond G, Petavy AF, Cambon M. Leishmaniasis in Auvergne (France). Med Mal Infect. 1985;15(3):107-9.

15. Naucke TJ, Menn B, Massberg D, Lorentz S. Sandflies and leishmaniasis in Germany. Parasitol Res. 2008;103 Suppl 1:65-8. doi:10.1007/s00436-008-1052-y.

16. Poeppl W, Obwaller AG, Weiler M, Burgmann H, Mooseder G, Lorentz S, et al. Emergence of sand flies (Phlebotominae) in Austria, a Central European country. Parasitol Res. 2013;112:4231-7. doi:10.1007/s00436-013-3615-9.

17. Zanet S, Sposimo P, Trisciuoglio A, Giannini F, Strumia F, Ferroglio E. Epidemiology of Leishmania infantum, Toxoplasma gondii, and Neospora caninum in Rattus rattus in absence of domestic reservoir and definitive hosts. Vet Parasitol. 2014;199:247-9.
18. Obwaller AG, Poeppl W, Naucke TJ, Luksch U, Mooseder G, Aspöck H, et al. Stable populations of sand flies (Phlebotominae) in Eastern Austria: comparison of the trapping seasons 2012 and 2013. Trends Entomol. 2014;10:49-53.

19. Theodor O. Psychodidae. Schweizerbart'sche Verlagsbuchhandlung. 1958;9:1-55.

20. Seccombe AK, Ready PD, Huddleston LM. A catalogue of Old World phlebotomine sand flies. Occas Pap Syst Entomol. 1993;8:1-60.

21. Toz SO, Culha G, Zeyrek FY, Ertabaklar H, Alkan MZ, Vardarlı AT, et al. A realtime ITS1-PCR based method in the diagnosis and species identification of Leishmania parasite from human and dog clinical samples in Turkey. PLoS Negl Trop Dis. 2013;7(5):e2205. doi:10.1371/journal.pntd.0002205.

22. el Tai NO, Osman OF, el Fari M, Presber W, Schönian G. Genetic heterogeneity of ribosomal internal transcribed spacer in clinical samples of Leishmania donovani spotted on filter paper as revealed by singlestrand conformation polymorphisms and sequencing. Trans R Soc Trop Med Hyg. 2000;94(5):575-9.

23. Kearse M, Moir R, Wilson A, Stones-Havas S, Cheung M, Sturrock S, et al. Geneious Basic: An integrated and extendable desktop software platform for the organization and analysis of sequence data. Bioinformatics. 2012; 28:1647-9.

24. Gaskin A, Schantz P, Jackson J, Birkenheuer A, Tomlinson L, Gramiccia M, et al. Visceral leishmaniasis in a New York foxhound kennel. J Vet Intern Med. 2002;16:34-44

25. Naucke TJ, Lorentz S. First report of venereal and vertical transmission of canine leishmaniosis from naturally infected dogs in Germany. Parasit Vectors. 2012:5:1-5. doi:10.1186/1756-3305-5-67.

26. Killick-Kendrick R. Phlebotomine vectors of the leishmaniases: a review. Med Vet Entomol. 1990:4:1-24.

27. Haouas N, Pesson B, Boudabous R, Dedet JP, Babba H, Ravel C. Development of a molecular tool for the identification of Leishmania reservoir hosts by blood meal analysis in the insect vectors. Am J Trop Med Hyg. 2007;77(6):1054-9.

28. Kamhawi S, Modi GB, Pimenta PF, Rowton E, Sacks DL. The vectorial competence of Phlebotomus sergenti is specific for Leishmania tropica and is controlled by species-specific, lipophosphoglycan-mediated midgut attachment. Parasitology. 2000;121(1):25-33.

29. Hlavacova J, Votypka J, Volf P. The effect of temperature on Leishmania (Kinetoplastida: Trypanosomatidae) development in sand flies. J Med Entomol. 2013;50(5):955-8.

30. Dostálová A, Volf P. Leishmania development in sand flies: parasite-vector interactions overview. Parasit Vectors. 2012:5(1):276. doi:10.1186/1756-3305-5-276.

31. Pimenta PFP, Modi GB, Pereira ST, Shahabuddin M, Sacks DL. A novel role for the peritrophic matrix in protecting Leishmania from the hydrolytic activities of the sand fly midgut. Parasitol. 1997;115(4):359-69.

32. Guimarães VC, Pruzinova K, Sadlova J, Volfova V, Myskova J, Filho SP, Volf P. Lutzomyia migonei is a permissive vector competent for Leishmania infantum. Parasit Vectors. 2016;9(1):159. doi:10.1186/s13071-016-1444-2.

33. Rossi E, Bongiorno G, El C, Di Muccio T, Scalone A, Gramiccia M, et al. Seasonal phenology, host-blood feeding preferences and natural Leishmania infection of Phlebotomus perniciosus (Diptera, Psychodidae) in a high-endemic focus of canine leishmaniasis in Rome province. Italy Acta Trop. 2008;105(2):158-65.

34. Poeppl W, Herkner H, Tobudic S, Faas A, Auer H, Mooseder G, et al. Seroprevalence and asymptomatic carriage of Leishmania spp. in Austria, a non-endemic European country. Clin Microbiol Infect. 2013;19(6):572-7.

35. Leschnik M, Löwenstein M, Edelhofer R, Kirtz G. Imported non-endemic, arthropod-borne and parasitic infectious diseases in Austrian dogs. Wien Klin Wochenschr. 2008;120(4):59-62. 